\title{
Susie Nanney
}

$\mathbf{O}$

n June 15, 2018, the nutrition community lost a colleague and friend, Susie Nanney. Susie was an associate professor at the University of Minnesota where she served as director of the Population Health Division. She was the founding director of the Health Equity in Policy Initiative for the Program in Health Disparities Research. Her research focused on obesity prevention, hunger relief, health disparities, and policy. She worked to improve children's and family health in underserved and rural communities, including in early care and education settings. We will miss her as a colleague and as a champion of promoting population health and reducing health disparities. 